\title{
The physical and chemical properties of planet forming disks
}

\author{
Inga Kamp ${ }^{1}$ \\ ${ }^{1}$ Kapteyn Astronomical Institute, University of Groningen, PO Box 800, 9700 AV Groningen,
} The Netherlands, email: kamp@astro.rug.nl

\begin{abstract}
VLT instruments and ALMA have revolutionized in the past five years our view and understanding of how disks turn into planetary systems. They provide exquisite insights into non-axisymmetric structures likely closely related to ongoing planet formation processes. The following cannot be a complete review of the physical and chemical properties of disks; instead I focus on a few selected aspects. I will review our current understanding of the physical properties (e.g. solid and gas mass content, snow and ice lines) and chemical composition of planet forming disks at ages of 1-few Myr, especially in the context of the planetary systems that are forming inside them. I will highlight recent advances achieved by means of consistent multi-wavelength studies of gas AND dust in protoplanetary disks.
\end{abstract}

Keywords. Astrochemistry; planetary systems: protoplanetary disks, formation; solar system: formation, comets, asteroids

\section{Introduction}

The physical properties have important consequences for the formation of planets such as how much 'solid' surface density is available in the various planet forming regions, the gravitational stability of disks, as well as the total disk gas mass available to keep planet formation ongoing at a few Myr. I will review here the icelines in disks - both from the modeling perspective as well as recent observational insights - and gas masses, mostly summarizing the current caveats and debates on the interpretation of CO rotational line observations and the prospects for using HD instead in future missions.

The chemical properties have implications for the composition of forming planets. The disk composition determines the composition of planetsimals and hence that of growing planetary cores, e.g. the water content as a function of distance from the star and also the $\mathrm{C} / \mathrm{O}$ ratio of planets and the composition of primary/secondary atmospheres. Two topics that received quite some attention in the past few years are the aspect of heritage versus in-situ for the chemical composition of planet forming disks and the role of (warm) surface chemistry for the 'solid' composition, ice and refractory material among them phyllosilicates; I focus here on these two.

These selected topics all demonstrate the close coupling between gas and dust in planet forming disks and its high relevance for the interpretation of observations. This coupling happens not only thermally through collisions, but also through grain opacities (e.g. non-thermal desorption processes), IR pumping for molecular excitation, gas pressure determining dust grain settling and migration, and varying effective grain surface area for chemical reactions throughout the disk. A proper interpretation of the rich existing and upcoming disk observations requires state-of-the-art disk models to include these coupling terms. 


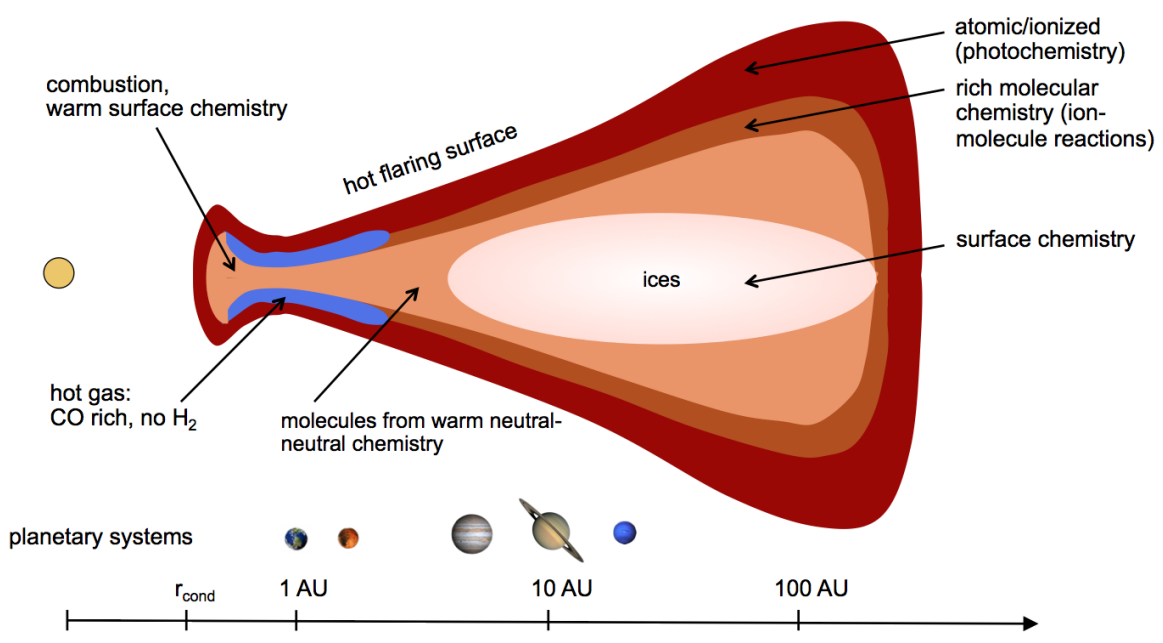

Figure 1. Chemical structure of a planet forming disk outlining the layered structure (atomic/molecular/ice) and the various types of chemistry (photochemistry, ion-molecule chemistry, neutral-neutral chemistry, combustion chemistry) relevant in the different parts of the disk.

\section{Disk structure}

Our knowledge of disk structure is largely based on seminal papers of steady state accretion disks (Pringle 1981) and irradiated disks (Kenyon \& Hartmann 1987; Chiang \& Goldreich 1997). Gas and dust temperatures decouple in the disk surface (Kamp \& Dullemond 2004; Jonkheid et al. 2004). While dust is mainly heated by the bulk luminosity of the central star, the gas thermal balance is driven by far-UV (FUV) and $\mathrm{X}$-rays from the central star, which are absorbed and scattered by dust grains in the disk surface (see Dullemond et al. 2007, for a review of disk structure). X-rays mainly interact with the gas that similarly absorbs and scatters them (Nomura et al. 2007; Aresu et al. 2011); if grains have grown much larger than the ISM, dust opacities become only important for high energy X-rays ( $E \gtrsim 5-10 \mathrm{eV}$, Rab et al. 2018).

Cosmic rays (CR) are less relevant for heating the gas, but they drive chemical processes close to the disk midplane such as non-thermal desorption and cosmic ray ionization. Disks are layered structures (e.g. Aikawa et al. 2002; Kamp \& Dullemond 2004, see Fig. 1) that can be divided into

(a) a hot ionized/atomic layer in which photochemistry dominates

(b) a warm molecular layer in which ion-molecule chemistry dominates

(c) a cold icy midplane in which CR ionization and surface chemistry dominate.

Surface chemistry encompasses a wide range of non-thermal desorption processes and has been reviewed recently by Cuppen et al. (2017). In addition to these three disk layers, Lee et al. (2010) discussed implications of combustion chemistry for the innermost parts of protoplanetary disks. A more general review of chemical evolution in disks including observations can be found in Bergin et al. (2007) and Dutrey et al. (2014).

Planet forming disks are shaped by dynamical processes such as accretion onto the central star, winds (radiation or magnetically driven), gas turbulence, vertical settling, collisional growth and radial migration of dust grains, as well as disk-planet interaction (e.g. Weidenschilling 1977; Miyake \& Nakagawa 1995; Dullemond \& Dominik 2004). 
Alexander (2008), Armitage (2011) and Birnstiel et al. (2016) provide recent reviews of the various processes.

Over the past decade, there has been increasing observational evidence that confirms the general picture described above. Avenhaus et al. (2018) found in their VLT/SPHERE survey of T Tauri disks that the scattered light surface displays a typical flaring structure; previously this had been traced with resolved PAH emission for the Herbig disk around HD 97048 (Lagage et al. 2006). In addition, dust settling has now been directly seen with ALMA. Mulders \& Dominik (2012) show that in a self-consistent settling model, mmsized dust grains are concentrated at heights of $z / r \lesssim 0.02$. Modeling the high spatial resolution HL Tau ALMA data (ALMA Partnership et al. 2015), Pinte et al. (2016) demonstrate that the similarity in gap width between major- and minor-axis requires a typical $z / r$ of $\lesssim 0.01$, consistent with a very low turbulence, $\alpha=3 \times 10^{-4}$.

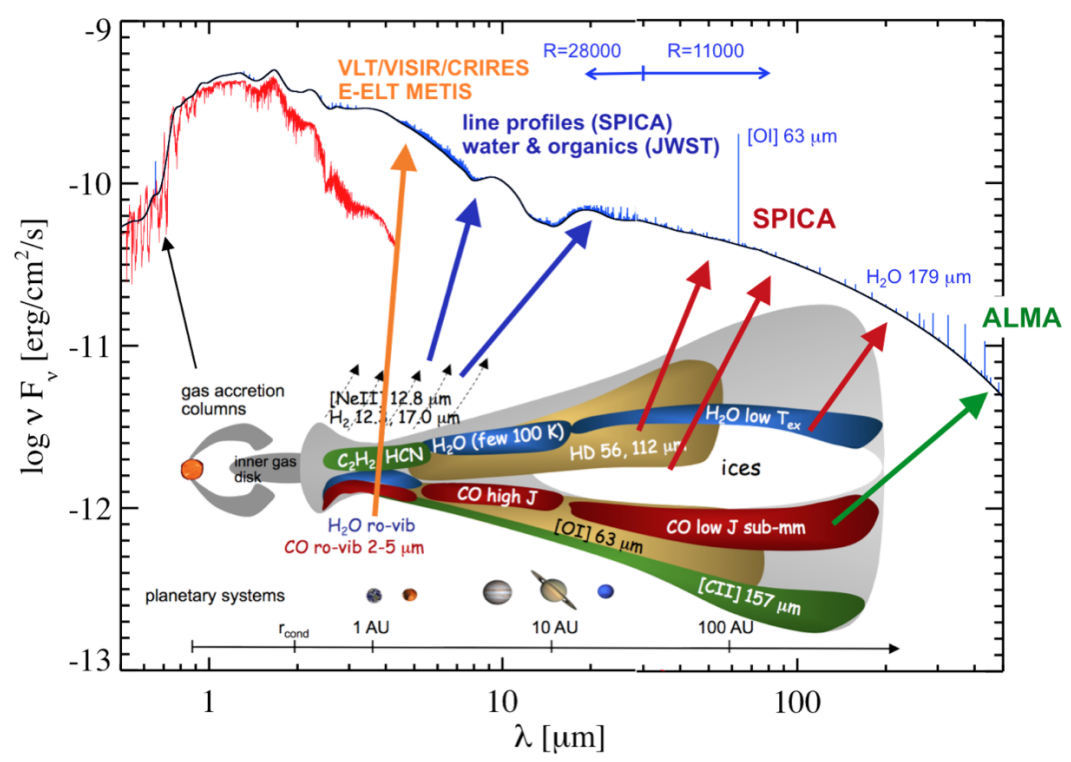

Figure 2. An overview of line emission from near-IR to submm wavelengths from planet forming disks and existing and upcoming instrumentation to detect it. The model SED is that of a typical $\mathrm{T}$ Tauri disk using two different spectral resolutions $(R=28000$ and $R=11000$ relevant for the planned SMI and SAFARI instruments onboard the proposed SPICA mission).

Understanding the physical conditions of the gas in these planet forming disks is challenging because gas lines emit according to their respective excitation conditions which depend to first order on the gas temperature, the density and the optical depth. Figure 2 illustrates how emission from hot gas in the inner disk occurs preferentially at near- to mid-IR wavelengths. For these lines, the dust optical depth at the corresponding wavelength determines how deep we can peer into these disks. Molecules such as CO and water, which possess a wide range of lines across the near- to far-IR wavelength range, can scan the surface and also probe successively deeper into the disk as the dust becomes more optically thin towards longer wavelengths. Since each line probes only a small region in such a planet forming disk, we need a suite of instruments to trace the gas across the entire disk such as VLT/VISIR and CRIRES (ELT/METIS in the future) for the hot (few 100 - few $1000 \mathrm{~K}$ ) gas at short wavelengths, JWST/NIRSPEC and MIRI in the near- to mid-IR, the proposed SPICA mission ranging from mid- to far-IR and bridging the gap to detecting cold (few $10 \mathrm{~K}$ ) gas with ALMA at submm wavelengths. 
We are now in an era where we have tens of disks with very rich multi-wavelengths and multi-kind observational data: X-ray to mm wavelengths and photometric, spectroscopic, spectro-astrometric, imaging and interferometric datasets for both gas and dust. Disk modeling has caught up with the challenge to interpret such complex datasets in the form of various radiation thermo-chemical models such as PRODiMo (Woitke et al. 2009; Kamp et al. 2010; Thi et al. 2011), DALI (Bruderer et al. 2012), and ANDES (Akimkin et al. 2013). The DIANA project has recently demonstrated for a sample of 14 objects that individual self-consistent parametrized dust+gas disk models are able to match the entirety of such complex datasets (Woitke et al. 2018, e.g. line fluxes across all wavelengths match observed ones to within a factor three). Such modeling can break the large degeneracies in the interpretation that often exist when only individual datasets are analysed.

\section{Disk physical properties}

These properties provide important constraints for planet formation models. The following paragraphs present two examples that received a lot of attention over the last few years, especially in the context of new ALMA observations: The location of icelines in disks and the determination of gas masses.

\subsection{Icelines}

Icelines in disks are not sharp transitions. Different binding energies for water on bare and icy grains as well as turbulent mixing lead to so-called 'snow borders' in which bare grains and icy grains coexist. Marseille \& Cazaux (2011) explore in their models the radial width of such 'snow borders' and find typical values of $0.03-0.2 \mathrm{au}$. Using a completely different modeling approach, Krijt et al. (2016) explore the vertical distribution of water vapor and ice in the presence of dust grain growth, settling and turbulent mixing. At low turbulence levels $\left(\alpha \leqslant 10^{-3}\right)$, the snowline resides about one pressure scale hight deeper in the disk compared to simple equilibrium models. Ciesla \& Cuzzi (2006) and Furuya \& Aikawa (2014) showed that — besides relocating snowlines — turbulent mixing and transport processes have large effects on the chemical composition of disks, especially the local abundances of water, ices and complex organic molecules. It is however notable that for example vertical column densities of gaseous CO in the outer disk ( $\gtrsim 50 \mathrm{au})$ are hardly affected by turbulent mixing; this confirms earlier results by Willacy et al. (2006).

At lower temperatures $(<100 \mathrm{~K})$, in the outer disk, photodesorption plays an important role in setting the height of the icelines. Ceccarelli \& Dominik (2005) postulated this effect to explain the high level of deuterated cold water emission in DM Tau; later Herschel/HIFI observations detected this "photodesorbed" water reservoir through the ground state lines of the main isotopologue $\mathrm{H}_{2} \mathrm{O}$ (Hogerheijde et al. 2011; Podio et al. 2013). Laboratory and thermo-chemical disk modeling efforts subsequently quantified the process (e.g. Fraser et al. 2011; Öberg et al. 2009; Kamp et al. 2013).

Very recently, Schwarz et al. (2016) and Pinte et al. (2018) directly observed the effect of photodesorption using ALMA CO isotopologue observations in the disks of TW Hya and IM Lup respectively. The CO temperature plateaus at $21 \mathrm{~K}$ at intermediate radii in both datasets; this is consistent with laboratory measurements of $\mathrm{CO}$ binding energies. In addition, the radial extent and location of CO as derived from the high spatial resolution channel maps can only be reproduced with models that take into account $\mathrm{CO}$ photodesorption (Pinte et al. 2018). The depth to which photons can penetrate these disks and drive photodesorption depends strongly on the local dust properties, demonstrating 
once more the high relevance of consistent dust and gas models in interpreting the new data.

\subsection{Gas masses}

For decades, many continuum surveys measured disk masses as a key input for the "planet forming potential" of these disks (e.g. Beckwith et al. 1990; Andre \& Montmerle 1994; Andrews \& Williams 2005; Andrews \& Williams 2007). Photometric measurements at long wavelength, where the emission is thought to be optically thin, have been used together with a representative dust emissivity (opacity) to quantify disk dust masses To probe how disks evolve into planetary systems, the gas-to-dust mass ratio was often taken as an indicator, primordial disks being rich in gas with a gas-to-dust ratio of $\sim 100$ and debris disks being gas-poor. Several gas surveys (e.g. FEPS, GASPS) aimed at deriving this key quantity for various star forming regions using tracers such as midIR line emission (warm gas) and $\mathrm{CO}$ submm lines (cold gas) and the [O I] $63 \mu \mathrm{m}$ fine structure line (e.g. Pascucci et al. 2006; Dent et al. 2013).

With the advent of more sensitive submm interferometers such as SMA and ALMA, Williams \& Best (2014) suggested to use the two rarer isotopologues ${ }^{13} \mathrm{CO}$ and $\mathrm{C}^{18} \mathrm{O}$ to determine cold gas masses in disks (diagnostic $\mathrm{CO}$ isotopologue mass diagram) and found gas-to-dust ratios below 20 in several Taurus disks. The work of Willacy et al. (2006) and Furuya \& Ailawa (2014) had shown that low levels of turbulence as detected in several planet forming disks (e.g. Flaherty et al. 2018, for TW Hya) should not affect the $\mathrm{CO}$ abundance in the outer disk. Miotello et al. (2016) show that isotope selective photodissociation can affect the gas measurements by an order of magnitude. Still ALMA surveys report gas-to-dust mass ratios well below 100 in 1-3 Myr old star forming regions (e.g. Miotello et al. 2017, for Lupus). Recently, Woitke et al. (2016) and Kamp et al. (2018) quantify the effects of changing dust properties and disk outer edges (tapering off) for the $\mathrm{CO}$ isotopologue ratio and the diagnostic $\mathrm{CO}$ isotopologue mass diagram. The DIANA multi-wavelength disk modeling project has shown that a sample of ten well known disks span over two orders of magnitude in $\mathrm{CO}$ isotopologue line fluxes, while the gas-to-dust mass ratio in the thermo-chemical dust+gas models, that are consistent with an even wider observational dataset, span gas-to-dust mass ratios between 50 and 450 (Kamp et al. 2018). It is important to note that the "main sequence" of CO isotopologue lines is consistently found in all disk modeling works (Williams \& Best 2014; Miotello et al. 2016; Kamp et al. 2018), but disk mass is not the only parameter shifting line fluxes up and down this main sequence; for example the maximum grain size or the power law index of the grain size distribution also play a key role. Using the now available multiband medium resolution ALMA surveys of several star forming regions, the underlying dust properties can be constrained observationally and consistent gas+dust modeling should be employed to derive better estimates of gas masses from $\mathrm{CO}$ isotopologue data. However, the statistics are often limited since many of the surveys are fairly shallow and the number of sources detected in both ${ }^{13} \mathrm{CO}$ and $\mathrm{C}^{18} \mathrm{O}$ remains small compared to the total number of sources detected in the dust continuum.

Since the HD abundance depends only on the primordial D abundance, an alternative to determine warm gas masses is the use of the two lowest excitation HD lines at 112 and $56 \mu \mathrm{m}\left(T_{\mathrm{ex}}=128.5\right.$ and $384.3 \mathrm{~K}$ respectively). Bergin et al. (2013) and later McClure et al. (2016) report HD masses or upper limits for seven T Tauri disks. Interestingly, the gas masses derived from the warm HD lines via disk modeling are often larger than

$\dagger$ Note that dust mass refers here to the solid mass in small dust grains up to mm-sizes; the solid mass in larger planetesimals and even protoplanets does not contribute significantly to the $\mathrm{mm}$ fluxes of disks and can hence not be measured this way. 
those derived via the CO lines. Trapman et al. (2017) use thermo-chemical disk models to explore the predictive power of these HD lines for disk gas masses and show that deep HD surveys with the proposed JAXA/ESA SPICA mission can detect disk gas masses down to $10^{-5} \mathrm{M}_{\odot}$ for typical $\mathrm{T}$ Tauri disks in nearby low mass star forming regions.

\section{Disk chemical properties}

These properties provide constraints for the composition of the planets forming within these disks. The following paragraphs review two controversial issues relating to these chemical properties: The question of heritage versus in-situ for the chemical composition of disks and the relevance of surface chemistry in disks.

\subsection{Heritage versus in-situ}

The thermal history of the material forming a disk is strongly affected by processes such as stellar outbursts, and grain growth during the collapse/disk formation phase. The collapse of a dense core is dynamically complex involving magnetic fields and possibly instabilities. A showcase example of this complexity is the ALMA high spatial resolution imaging of the binary protostar system IRAS 16293-2422 (PILS, Jørgensen et al. 2016).

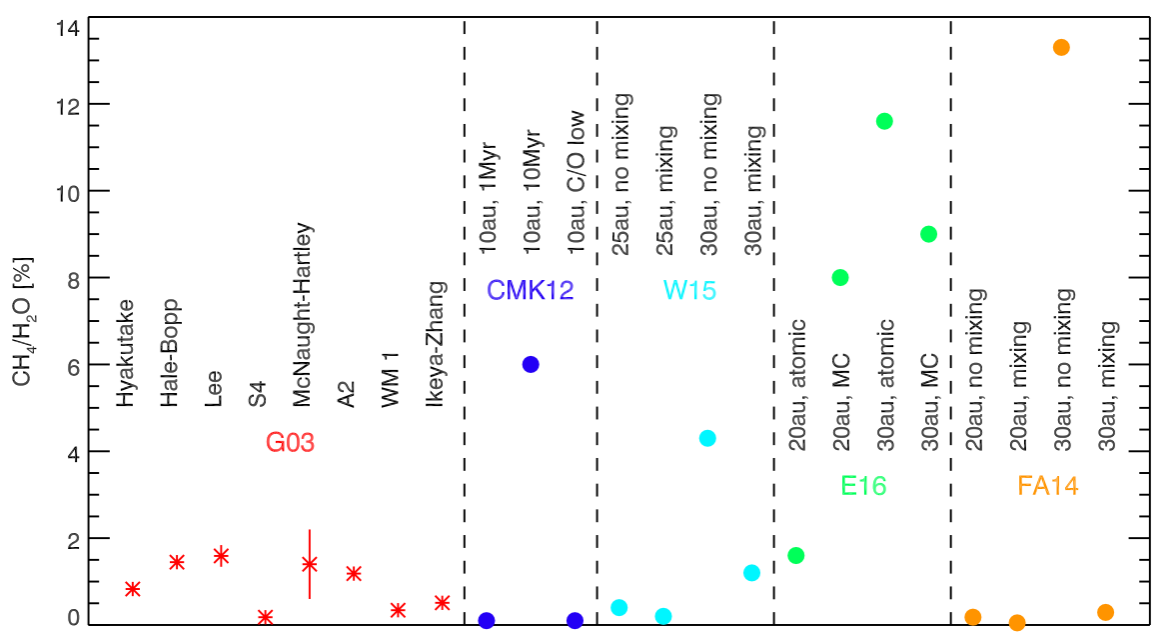

Figure 3. Comparsion between a collection of methane/water ice abundance ratios (with error bars) in Oort cloud comets (Gibb et al. 2003) and a sample of disk models: Chaparro et al. (2012, blue, $\mathrm{C} / \mathrm{O}=0.45$, low $\mathrm{C} / \mathrm{O}=0.16)$, Willacy et al. (2015, cyan), Eistrup et al. (2016, green), and Furuya \& Aikawa (2014, orange). Figure updated from a previous version by E. Gibb (private communication).

Several works studied the ice processing during the disk formation phase (e.g. Chick \& Cassen 1997; Visser et al. 2009; Visser et al. 2011; Furuya et al. 2012; Drozdovskaya et al. 2014). The comparison of comets and outer disks composition can provide important clues as to the amount of additional processing within the disk and the thermal history of ices. Willacy et al. (2015) show that most $(\mathrm{D} / \mathrm{H})_{\text {water }}$ measurements are only compatible with disk models that include mixing processes. Cleeves et al. (2014) go one step further using a comprehensive range of disk ionisation sources and a detailed chemical model of the protosolar disk to claim that a significant fraction of HDO in the disk stems from accretion and inheritance of interstellar ices. Figure 3 shows a comparison between the methane/water abundance ratio in cometary ices and the same ratio in a series of disk 
models. It becomes clear that this ice ratio depends strongly on the initial condition (atomic versus molecular), the elemental abundances (not only $\mathrm{C}$ and $\mathrm{O}$, but also other metals as electron sources), mixing, as well as the chemical age of the disk. The nomixing models and the young chemical ages give a better agreement with the observed $\mathrm{CH}_{4} / \mathrm{H}_{2} \mathrm{O}$ ratio than mixing/old models (contrary to $\mathrm{D} / \mathrm{H}$ ). The initial composition (atomic or molecular cloud values) as well as the element abundances $(\mathrm{C} / \mathrm{O})$ play a large role in setting the ice abundances in the outer disk (beyond $10 \mathrm{au}$ ). However, we are only at the beginning since many other effects also impact the ice composition, especially also the relative abundances of ices, such as layering and diffusion within ices, grain growth and migration, and the role of irradiation within ices.

\section{2. (Warm) Surface chemistry}

Surface chemistry itself is less well understood compared to gas-phase chemistry and the status and current limitations are comprehensively reviewed by Cuppen et al. (2017). Surface chemistry is often invoked to explain the formation of COMs (complex organic molecules with six or more atoms, at least two of which are C, N, O). Ciesla \& Sandford (2012) form COMs via mixing of icy grains in disks, subsequently exposing those icy grains to different levels of UV dosage. Drozdovskaya et al. (2016) propose that the abundances of COMs are strongly affected by the dynamical history of the disk build-up evolution (pure infall versus infall+viscous spreading). Walsh et al. (2014) show that even without taking details of disk build-up into account, significant abundances of COMs can be synthesized in disks through grain surface chemistry.

But also warm surface chemistry has recently received more attention in studying the composition of the inner few au as well and the link to terrestrial planets and asteroids. Combustion chemistry can burn carbonaceous dust inside $\sim 1$ au (e.g. Lee et al. 2010; Anderson et al. 2017), although the level of carbon depletion may be limited by the efficiency of radial and vertical mixing processes, which replenish carbon rich material faster than burning can proceed (Klarmann et al. 2018).

A second process driven by warm surface chemistry is the transformation of silicates into phyllosilicates (sheet silicates with intercalated water). Early work by Fegley \& Prinn (1989) dismissed the relevance of this process based on the long timescales it required compared to the lifetime of disks. More recent works by Stimpfl et al. (2006) and Muralidharan et al. (2008) using realistic silicate surfaces and adsorption energies find higher efficiencies in the solar nebula around the region where Earth has formed. We recently revisited these processes using both Monte Carlo simulations to assess the efficiency of water adsorption under high temperature/pressure conditions inside $\sim 1$ au (D'Angelo et al. 2018) and the subsequent surface chemistry that transforms part of the silicate grains into phyllosilcate material (Thi et al. 2018). Inside the water snowline, this process can proceed within $1 \mathrm{Myr}$ to produce grains that contain a significant fraction of water (up to a few \%). If the process occurs before grains have substantially grown, this is an alternative pathway to incorporate water into the proto-Earth, i.e. contribute to the water content within the Earth mantle.

\section{Outlook}

The future has great prospects to employ disk observations in conjunction with detailed disk models (radiation, physics, chemistry, dynamics) to achieve a more comprehensive view on planet formation. ALMA and VLT (now and in the future) will provide molecular channel maps to confront thermo-chemical disk models (gas+dust), to characterize rings and non-axisymmetric gas structures (embedded protoplanets, vortices), to de- 
tect circumplanetary disks, and study inner disk dispersal processes. In the near future, SOFIA/HIRMES $(\sim 2019)$ will detect thermal ice features and HD in a about a dozen disks showcasing the potential of such data. From 2021 onwards, JWST will provide data to study the warm gas+dust composition/evolution in the inner $\sim 10 \mathrm{au}$. ELT/METIS (beyond $\sim 2025$ ) will provide detailed kinematics (channel maps) in the inner $\sim 10$ au of disks, and study the formation and properties of circumplanetary disks. Further ahead beyond 2030, the proposed SPICA mission can study the trail of water, ice and solids during the entire planet formation phase through large unbiased surveys of water, HD, ices, and dust mineralogy well into the debris disk stage.

\section{References}

Aikawa, Y., van Zadelhoff, G. J., van Dishoeck, E. F., \& Herbst, E. 2002, A $\mathscr{E} A, 386,622$

Akimkin, V., Zhukovska, S., Wiebe, D., et al. 2013, ApJ, 766, 8

Alexander, R. 2008, NewAR, 52, 60

ALMA Partnership, Brogan, C. L., Pérez, L. M., et al. 2015, ApJL, 808, L3

Anderson, D. E., Bergin, E. A., Blake, G. A., et al. 2017, ApJ, 845, 13

Andre, P. \& Montmerle, T. 1994, ApJ, 420, 837

Andrews, S. M. \& Williams, J. P. 2005, ApJ, 631, 1134

Andrews, S. M. \& Williams, J. P. 2007, ApJ, 671, 1800

Aresu, G., Kamp, I., Meijerink, R., et al. 2011, A\&A, 526, A163

Armitage, P. J. 2011, Annu. Rev. Astron. Astrophys., 49, 195

Avenhaus, H., Quanz, S. P., Garufi, A., et al. 2018, ApJ, 863, 44

Beckwith, S. V. W., Sargent, A. I., Chini, R. S., \& Guesten, R. 1990, AJ, 99, 924

Bergin, E. A., Aikawa, Y., Blake, G. A., \& van Dishoeck, E. F. 2007, Protostars and Planets V, 751

Bergin, E. A., Cleeves, L. I., Gorti, U., et al. 2013, Nature, 493, 644

Birnstiel, T., Fang, M., \& Johansen, A. 2016, SSRv, 205, 41

Bruderer, S., van Dishoeck, E. F., Doty, S. D., \& Herczeg, G. J. 2012, A\&̛A, 541, A91

Ceccarelli, C. \& Dominik, C. 2005, A\& A, 440, 583

Chaparro Molano, G. \& Kamp, I. 2012, A\&A, 537, A138

Chiang, E. I. \& Goldreich, P. 1997, ApJ, 490, 368

Chick, K. M. \& Cassen, P. 1997, ApJ, 477, 398

Ciesla, F. J. \& Cuzzi, J. N. 2006, Icarus, 181, 178

Ciesla, F. J. \& Sandford, S. A. 2012, Science, 336, 452

Cleeves, L. I., Bergin, E. A., Alexander, C. M. O. ., et al. 2014, Science, 345, 1590

Cuppen, H. M., Walsh, C., Lamberts, T., et al. 2017, SSRv, 212, 1

D’Angelo, M., Cazaux, S., Kamp, I., Thi, W.-F., \& Woitke, P. 2018, ArXiv e-prints

Dent, W. R. F., Thi, W. F., Kamp, I., et al. 2013, PASP, 125, 477

Drozdovskaya, M. N., Walsh, C., van Dishoeck, E. F., et al. 2016, MNRAS, 462, 977

Drozdovskaya, M. N., Walsh, C., Visser, R., Harsono, D., \& van Dishoeck, E. F. 2014, MNRAS, 445, 913

Dullemond, C. P. \& Dominik, C. 2004, A\& A, 421, 1075

Dullemond, C. P., Hollenbach, D., Kamp, I., \& D'Alessio, P. 2007, Protostars and Planets V, 555

Dutrey, A., Semenov, D., Chapillon, E., et al. 2014, Protostars and Planets VI, 317

Eistrup, C., Walsh, C., \& van Dishoeck, E. F. 2016, A 6 A, 595, A83

Fegley, Jr., B. \& Prinn, R. G. 1989, in The Formation and Evolution of Planetary Systems, ed. H. A. Weaver \& L. Danly, 171-205

Flaherty, K. M., Hughes, A. M., Teague, R., et al. 2018, ApJ, 856, 117

Fraser, H. J., Collings, M. P., McCoustra, M. R. S., \& Williams, D. A. 2001, MNRAS, 327, 1165

Furuya, K. \& Aikawa, Y. 2014, ApJ, 790, 97

Furuya, K., Aikawa, Y., Tomida, K., et al. 2012, ApJ, 758, 86 
Gibb, E. L., Mumma, M. J., Dello Russo, N., DiSanti, M. A., \& Magee-Sauer, K. 2003, Icarus, 165,391

Hogerheijde, M. R., Bergin, E. A., Brinch, C., et al. 2011, Science, 334, 338

Jonkheid, B., Faas, F. G. A., van Zadelhoff, G.-J., \& van Dishoeck, E. F. 2004, A\&JA, 428, 511

Jørgensen, J. K., van der Wiel, M. H. D., Coutens, A., et al. 2016, A\&\&A, 595, A117

Kamp, I., Antonellini, S., Carmona, A., Ilee, J., \& Rab, C. 2018, in The Cosmic Wheel and the Legacy of the AKARI Archive: From Galaxies and Stars to Planets and Life, ed. T. Ootsubo, I. Yamamura, K. Murata, \& T. Onaka, 89-96

Kamp, I. \& Dullemond, C. P. 2004, ApJ, 615, 991

Kamp, I., Thi, W.-F., Meeus, G., et al. 2013, A\&A, 559, A24

Kamp, I., Tilling, I., Woitke, P., Thi, W., \& Hogerheijde, M. 2010, A\&A, 510, A260000

Kenyon, S. J. \& Hartmann, L. 1987, ApJ, 323, 714

Klarmann, L., Ormel, C. W., \& Dominik, C. 2018, A\& A, 618, L1

Krijt, S., Ciesla, F. J., \& Bergin, E. A. 2016, ApJ, 833, 285

Lagage, P.-O., Doucet, C., Pantin, E., et al. 2006, Science, 314, 621

Lee, J.-E., Bergin, E. A., \& Nomura, H. 2010, ApJL, 710, L21

Marseille, M. G. \& Cazaux, S. 2011, A\&A, 532, A60

McClure, M. K., Bergin, E. A., Cleeves, L. I., et al. 2016, ApJ, 831, 167

Miotello, A., van Dishoeck, E. F., Kama, M., \& Bruderer, S. 2016, A\& A, 594, A85

Miotello, A., van Dishoeck, E. F., Williams, J. P., et al. 2017, A\&SA, 599, A113

Miyake, K. \& Nakagawa, Y. 1995, ApJ, 441, 361

Mulders, G. D. \& Dominik, C. 2012, A\&A, 539, A9

Muralidharan, K., Deymier, P., Stimp, M., de Leeuw, N. H., \& Drake, M. J. 2008, Icarus, 198, 400

Nomura, H., Aikawa, Y., Tsujimoto, M., Nakagawa, Y., \& Millar, T. J. 2007, ApJ, 661, 334

Öberg, K. I., Linnartz, H., Visser, R., \& van Dishoeck, E. F. 2009, ApJ, 693, 1209

Pascucci, I., Gorti, U., Hollenbach, D., et al. 2006, ApJ, 651, 1177

Pinte, C., Dent, W. R. F., Ménard, F., et al. 2016, ApJ, 816, 25

Pinte, C., Ménard, F., Duchêne, G., et al. 2018, A\& $A$, 609, A47

Podio, L., Kamp, I., Codella, C., et al. 2013, ApJL, 766, L5

Pringle, J. E. 1981, Annu. Rev. Astron. Astrophys., 19, 137

Rab, C., Güdel, M., Woitke, P., et al. 2018, A\& $\&$, 609, A91

Schwarz, K. R., Bergin, E. A., Cleeves, L. I., et al. 2016, ApJ, 823, 91

Stimp, M., Walker, A., Drake, M., de Leeuw, N., \& Deymier, P. 2006, Journal of Crystal Growth, 294,83

Thi, W. F., Hocuk, S., Kamp, I., et al. 2018, arXiv e-prints

Thi, W.-F., Woitke, P., \& Kamp, I. 2011, MNRAS, 412, 711

Trapman, L., Miotello, A., Kama, M., van Dishoeck, E. F., \& Bruderer, S. 2017, A\&A, 605, A69

Visser, R., Doty, S. D., \& van Dishoeck, E. F. 2011, A\& A, 534, A132

Visser, R., van Dishoeck, E. F., \& Black, J. H. 2009, A\&A, 503, 323

Walsh, C., Millar, T. J., Nomura, H., et al. 2014, A\&SA, 563, A33

Weidenschilling, S. J. 1977, MNRAS, 180, 57

Willacy, K., Alexander, C., Ali-Dib, M., et al. 2015, SSRv, 197, 151

Willacy, K., Langer, W., Allen, M., \& Bryden, G. 2006, ApJ, 644, 1202

Williams, J. P. \& Best, W. M. J. 2014, ApJ, 788, 59

Woitke, P., Kamp, I., Antonellini, S., et al. 2018, arXiv e-prints

Woitke, P., Kamp, I., \& Thi, W.-F. 2009, A\& A, 501, 383

Woitke, P., Min, M., Pinte, C., et al. 2016, A\&AA, 586, A103

\section{Discussion}

MAROv: What mechanism of dust particles integration do you postulate in your model whether you believe it could be by integration per se through a direct collision mechanism, or rather some interaction process involving, say, original dust cluster formation is to be 
invoked? In my mind the latter would significantly ease the integration and particle growth process, which is supported by the results of my team computer modeling.

KAMP: First of all, I myself have no model of dust particle growth. Growth is now already detected at very early stages of star formation, e.g. coreshine. The very first condensation of dust is indeed thought to proceed via clusters that form e.g. around AGB stars. However, similar processes can occur if refractory material sublimates in disks close to the star due to e.g. outbursts and then recondenses. 